\title{
Rickettsia felis: molecular characterization of a new member of the spotted fever group
}

\author{
Donald H. Bouyer, ${ }^{1}$ John Stenos, ${ }^{2}$ Patricia Crocquet-Valdes, ${ }^{1}$ \\ Cecilia G. Moron, ${ }^{1}$ Vsevolod L. Popov, ${ }^{1}$ Jorge E. Zavala-Velazquez, ${ }^{3}$ \\ Lane D. Foil, ${ }^{4}$ Diane R. Stothard, ${ }^{5}$ Abdu F. Azad ${ }^{6}$ and David H. Walker ${ }^{1}$
}

Author for correspondence: David H. Walker. Tel: +1 409772 2856. Fax: +1 4097722500.

e-mail: dwalker@utmb.edu

\footnotetext{
1 Department of Pathology, WHO Collaborating Center for Tropical Diseases, University of Texas Medical Branch, 301 University Blvd, Galveston, TX 77555-0609, USA

2 Australian Rickettsial Reference Laboratory, Douglas Hocking Medical Institute, Geelong Hospital, Geelong, Australia

3 Department of Tropical Pathology, Universidad Autonoma de Yucatan, Merida, Yucatan, Mexico

4 Department of Entomology, LSU Agricultural Center, Baton Rouge, LA 70803-1710, USA

5 Division of Infectious Diseases, Indiana University School of Medicine, Indianapolis, IN 46202, USA

${ }^{6}$ Department of Microbiology and Immunology, University of Maryland School of Medicine, Baltimore, MD 21201, USA
}

In this report, placement of Rickettsia felis in the spotted fever group (SFG) rather than the typhus group (TG) of Rickettsia is proposed. The organism, which was first observed in cat fleas (Ctenocephalides felis) by electron microscopy, has not yet been reported to have been cultivated reproducibly, thereby limiting the standard rickettsial typing by serological means. To overcome this challenge, several genes were selected as targets to be utilized for the classification of $R$. felis. DNA from cat fleas naturally infected with $R$. felis was amplified by PCR utilizing primer sets specific for the $190 \mathrm{kDa}$ surface antigen (rOmpA) and $17 \mathrm{kDa}$ antigen genes. The entire 5513 bp rompA gene was sequenced, characterized and found to have several unique features when compared to the rompA genes of other Rickettsia. Phylogenetic analysis of the partial sequence of the $17 \mathrm{kDa}$ antigen gene indicated that $R$. felis is less divergent from the SFG rickettsiae than from the TG rickettsiae. The data corroborate results from previous reports that analysed the citrate synthase, $16 S$ rRNA, rompB (135 kDa surface antigen), metK, fts $Y$, polA and dnaE genes that placed $R$. felis as a member of the SFG. The organism is passed transstadially and transovarially, and infection in the cat flea has been observed in the midgut, tracheal matrix, muscle, hypodermis, ovaries and testes.

Keywords: Rickettsia felis, rickettsial outer-membrane protein A, 17 kDa gene, Ctenocephalides felis, tandem repeat domain

\section{INTRODUCTION}

In 1990, during a study investigating potential vectors for Ehrlichia risticii, rickettsia-like organisms were observed in the midgut epithelial cells of adult cat fleas, Ctenocephalides felis, by electron microscopy (Adams et al., 1990). The organisms were found only in a group of fleas obtained from El Labs, from which the original designation of the organism (ELB) was derived, and not in any of the other three sources of fleas. The

Abbreviations: CS, citrate synthase; SFG, spotted fever group; TG, typhus group.

The GenBank accession numbers for the $17 \mathrm{kDa}$ protein gene and rompA sequences of Rickettsia felis are AF195118 and AF191026, respectively. organisms were described as having an ultrastructure and infection pattern similar to that of Rickettsia typhi. The organisms were $0 \cdot 25-0 \cdot 45 \mu \mathrm{m}$ in diameter by $1.5 \mu \mathrm{m}$ in length and were found not only in the midgut, but also in the tracheal matrix, muscles and reproductive tissues of the fleas. The organisms contained trilaminar cell walls that were characteristic of rickettsiae with a well-defined inner cell membrane and outer membrane. Measurements of the microcapsular layer, outer and inner leaflets of the outer membrane, and the periplasmic space strongly resembled those in other Rickettsia species. The first attempts to characterize the organism involved the amplification of the $17 \mathrm{kDa}$ antigen, citrate synthase (CS) and $190 \mathrm{kDa}$ antigen (rompA) genes (Azad et al., 1992). The ELB 
agent was found to be distinguishable from $R$. typhi by RFLP analysis of the $17 \mathrm{kDa}$ gene product digested with $A$ haII or $A l u \mathrm{I}$. The CS gene was used to confirm that the organism found in the cat fleas was the ELB agent and not $R$. typhi, which can also be found occasionally in these fleas. The RFLP pattern of the CS gene amplified from the ELB agent in cat fleas differed from that of $R$. typhi. Attempts at that time to amplify the romp $A$ gene from the ELB agent proved to be unsuccessful. It has also been shown by two experimental observations that the ELB agent can be transmitted trans-stadially and transovarially. Unfed cat fleas that were negative by PCR for the ELB agent tested positive for the $17 \mathrm{kDa}$ protein gene of the ELB agent after feeding on infected cats (Azad et al., 1992). Also, the ELB agent was present in freshly deposited eggs as determined by PCR (Azad et al., 1992). The first serological assays for the ELB agent were also conducted in this study. Antisera and mAbs generated against $R$. typhi were used to examine smears of newly emerged fleas from both the El Labs and negative controls. Indirect immunofluorescent staining detected the ELB agent in the sample fleas, but not in the control fleas. In surveys of fleas in Los Angeles County, $\mathrm{CA}$ and in Texas, the $17 \mathrm{kDa}$ and CS genes were used to investigate the natural occurrence of the ELB agent (Williams et al., 1992; Schriefer et al., 1994a). The results from both studies indicated that infection of the cat flea with the ELB agent is more prevalent than with $R$. typhi. One study reported an infection rate of $3.8 \%$ for the ELB agent (Schriefer et al., 1994a). This investigation also detected the ELB rickettsia within the spleen of opossums, thus suggesting that this animal might play a role in the maintenance of the ELB agent in nature and proving the capacity of the rickettsia to cause disseminated infection. The ELB agent has been identified in flea colonies from various regions of the United States through the use of RFLP analysis of PCR products of the $17 \mathrm{kDa}$ and CS genes (Higgins et al., 1996). Analysis of the eight colonies showed that they were infected with the ELB agent with a range of prevalence within each colony of $43-93 \%$. The possible source of ELB in these colonies was subsequently traced to the El Labs, which provided fleas as starter stock or to replenish the colony. Attempts to infect mammalian cells and SCID mice with the ELB agent were not successful. Two publications are based upon the study of organisms considered to represent ELB agent propagated in cell culture (Radulovic et al., 1995a, b); however, the cultured agent could not be reproducibly propagated and maintained in further culture. In addition, there is the possibility of contamination of the later passages of the culture with $R$. typhi, which has also been found to infect the cat flea. Data from these two reports are not included in the evidence included here that the ELB agent is a novel Rickettsia species. In 1996, it was proposed that the ELB agent be designated Rickettsia felis in recognition of its discovery and origin in the cat flea (Higgins et al., 1996). Subsequent additions to our knowledge of $R$. felis have used this name in the biomedical and scientific literature (Noden et al., 1998; Andersson \& Andersson, 1999; Andersson et al., 1999; Bouyer et al., 1999).

There have also been reports implicating the involvement of $R$. felis in human disease, indicating its potential importance as a newly emerging pathogen (Schriefer et al., 1994a; Zavala-Velazquez et al., 2000). Since $R$. felis has thus far resisted attempts at cultivation, thereby limiting the standard rickettsial typing by serological means, the organism was further characterized through the utilization of molecular means as has been employed to describe other uncultivated rickettsial organisms (Anderson et al., 1992; Neibylski et al., 1997). The rompA gene from cat fleas containing $R$. felis was amplified by PCR using different primers and amplification conditions and sequencing data for the $17 \mathrm{kDa}$ protein gene were obtained. The rompA gene was selected as one of our target genes based on the fact that only spotted fever group (SFG) rickettsiae contain this gene and that the proof of the existence of this gene in $R$. felis would have a significant impact upon its classification (Roux et al., 1996). In this study, the successful sequencing and characterization of the rompA gene of $R$. felis is reported. These data, along with other sequence information on the CS, $\operatorname{romp} B, 17 \mathrm{kDa}$ protein, met $K$, fts $Y$, polA and dnaE genes, are the justification for describing $R$. felis as a member of the SFG.

\section{METHODS}

Source of infected fleas. The infected cat fleas were maintained in the laboratory colony of the Department of Entomology, Louisiana State University Agricultural Center (Henderson \& Foil, 1993).

DNA isolation. A pool of infected cat fleas was washed with sterile PBS (137 mM sodium chloride, $2.7 \mathrm{mM}$ potassium chloride, $4.3 \mathrm{mM}$ dibasic sodium phosphate, $1.4 \mathrm{mM}$ potassium phosphate) and homogenized. The homogenate was resuspended in $1 \mathrm{ml}$ PBS containing $1 \%$ SDS and $1 \%$ proteinase $\mathrm{K}$, placed in a $1.5 \mathrm{ml}$ microcentrifuge tube and incubated for $1 \mathrm{~h}$ at $37^{\circ} \mathrm{C}$ followed by a $10 \mathrm{~min}$ incubation at $56{ }^{\circ} \mathrm{C}$. A series of phenol/chloroform extractions was performed on the homogenate followed by a single chloroform extraction. The DNA was ethanol-precipitated and dried, and the pellet was resuspended in sterile water.

PCR. Amplification of an aliquot of the DNA extracted from fleas using the outer primers for the Rickettsia $17 \mathrm{kDa}$ gene was performed as described previously (Webb et al., 1990; Schriefer et al., 1994b; Higgins et al., 1996). Six micrograms of DNA in a $5 \mu$ l volume of DNA extract was added to $1 \times$ PCR buffer (Boehringer Mannheim), $200 \mu \mathrm{M}$ nucleotide mixture, 10 pmol each primer, 1.25 units Taq polymerase (Boehringer Mannheim) and distilled water to a final volume of $100 \mu \mathrm{l}$. The PCR was performed in a DNA Thermal Cycler (Perkin-Elmer Cetus). The PCR product from this reaction was further amplified using a set of nested primers for the $17 \mathrm{kDa}$ gene (Higgins et al., 1996). PCR conditions for both reactions were as previously described (Webb et al., 1990; Schriefer et al., 1994b; Higgins et al., 1996).

Genome walking. One region of the $R$. felis romp $A$ gene, the promoter and ATG start site, was amplified by the utilization 
Table 1 Primers for amplification of $R$. felis rompA gene

\begin{tabular}{|c|c|c|}
\hline Primer & Sequence & Reference \\
\hline \multicolumn{3}{|c|}{ Promoter region and ATG start site } \\
\hline GSP1 (RF321-292) & AGCTCCTCCCGTATCTACCACTGAACCTAA & This study \\
\hline ASP1 & GTAATACGACTCACTATAGGGC & Invitrogen \\
\hline GSP1 (RF65-38r) & AGCTCCTCCCGTATCTACCACTGAACCTAA & This study \\
\hline ASP2 & ACTATAGGGCACGCGTGGT & Invitrogen \\
\hline \multicolumn{3}{|c|}{ (1) } \\
\hline $190 \cdot 70$ & ATGGCGAATAATTCTCCAAAA & Regnery et al. (1991) \\
\hline $190 \cdot 602 n$ & AGTGCAGCAATTCGCTCCCCCT & \\
\hline \multicolumn{3}{|c|}{$5^{\prime}$ repeat region overlap } \\
\hline Rf $247 f$ & AATAATTTTGCAGCAGGTCTTT & This study \\
\hline Rf Repeat $r$ & TGACTCAATGCTCCACTTTAGAT & \\
\hline \multicolumn{3}{|l|}{ Repeat region } \\
\hline 675 & CCAGACAGATGCTGCCATTAAGC & Walker et al. (1995) \\
\hline 2940 & TTCCGATCTAGACTTCCTCCAAGC & \\
\hline \multicolumn{3}{|l|}{ Repeat $3^{\prime}$ overlap } \\
\hline Rf-Repeat $f$ & AGGCGGTGATAATGTAGGTGTCT & This study \\
\hline RfB5-27R & TTACTCGCAGCTCCAAAATCTAT & \\
\hline \multicolumn{3}{|l|}{$3^{\prime}$ region $(1.4 \mathrm{~kb})$} \\
\hline RR-3622f & GCTGGAGGAAGCCTAGCTGCG & This study \\
\hline RR-4999r & TGACCAACCGAATTAGCCGC & \\
\hline \multicolumn{3}{|l|}{$3^{\prime}$ region $(1 \cdot 2 \mathrm{~kb})$} \\
\hline F3-4936 & GGTGGTCAGGCTCTGAAGCTAAAAC & Stenos \& Walker (2000) \\
\hline B21-6324 & TGCAGTTTGATAACCGACAGTCTC & \\
\hline \multicolumn{3}{|l|}{$3^{\prime}$ region $(1.0 \mathrm{~kb})$} \\
\hline 6049 & ACTGGTGGCACTATAGGTTTTGAC & P. A. Crocquet-Valdes, \\
\hline 7019 & ATCGGCAGTTTTTCTAATAATAAT & personal communication \\
\hline
\end{tabular}

of a Genome Walker kit (Clontech) with a few modifications of the manufacturer's instructions. The primers are listed in Table 1. The DNA extract from the fleas was divided into five aliquots and subjected to digestion by five blunt-endcutting restriction enzymes (DraI, EcoRV, PvuII, ScaI, StrI) at $37^{\circ} \mathrm{C}$ overnight. The reaction mixtures were subjected to a series of phenol/chloroform extractions followed by chloroform extraction and ethanol precipitation. Adapters from the kit were ligated to the digested DNA samples at $16{ }^{\circ} \mathrm{C}$ overnight. The reaction was stopped by incubating the samples at $70^{\circ} \mathrm{C}$ for $5 \mathrm{~min}$, followed by the addition of Tris/EDTA buffer $(10 \mathrm{mM}$ Tris/ $\mathrm{HCl}, 1 \mathrm{mM}$ EDTA, pH $8 \cdot 0)$. Each sample was PCR-amplified in the primary reaction using the Advantage Tth polymerase kit (Clontech) with the primary $R$. felis romp $A$ gene specific primer (GSP1) and adapter primer 1 (AP1) from the kit. The nested PCR was performed as above with the addition of $1 \mu l$ of the primary reactions as the template and the substitution of nested primers GSP2 and AP2 for the GSP1 and AP1 primers. The cycling parameters utilized were modified from those proposed in the manufacturer's instructions with the annealing temperature being $3{ }^{\circ} \mathrm{C}$ higher than that given in the instructions. The sequences of the primers are listed in Table 1.

Sequencing of the rompA gene. DNA extracts from the infected cat fleas were utilized for sequencing the $R$. felis romp $A$ gene. Primers designed for amplification of the repeat region and $5^{\prime}$ region of the gene were from published sequences or were generated from sequence analysis of the
R. felis PCR products (Table 1). The PCR amplification conditions for the reactions were 1 cycle at $95^{\circ} \mathrm{C}$ for $5 \mathrm{~min}$, 30 cycles of $1 \mathrm{~min}$ at $95^{\circ} \mathrm{C}, 20 \mathrm{~s}$ at $48^{\circ} \mathrm{C}, 3 \mathrm{~min}$ at $72^{\circ} \mathrm{C}$ followed by 1 cycle of $5 \mathrm{~min}$ at $72^{\circ} \mathrm{C}$ and 1 soak cycle at $4{ }^{\circ} \mathrm{C}$. An aliquot of the PCR products was analysed by resolution on $1.3 \%(\mathrm{w} / \mathrm{v})$ agarose gels that were stained with ethidium bromide and visualized by a UV light source. The remaining PCR products were cloned using the TOPO TA Cloning kit (Invitrogen) and plated on selective media containing ampicillin and X-Gal/IPTG overnight at $37^{\circ} \mathrm{C}$. Positive clones were selected and grown in LB medium containing ampicillin. Plasmid DNA was isolated using the High Pure Plasmid Isolation kit (Roche Molecular Biochemicals) and digested with EcoRI according to the manufacturer's instructions. The restriction enzyme digests were analysed in a $1.3 \%(\mathrm{w} / \mathrm{v})$ agarose gel. Plasmids that contained DNA inserts were sequenced twice using an ABI automated sequencer with M13 and T7 sequencing primers (Gibco-BRL).

Genetic analysis. The primer design sequence alignment and preliminary comparison were facilitated through the use of the software programs GCG (Wisconsin Package, version 10.0, Genetics Computer Group, Madison, WI) and LASERGENE (DNASTAR, Madison, WI), which are built upon the CLUSTAL algorithm platform (Higgins \& Sharp, 1989). The percentages of similarity were determined by the CLUSTAL method.

Phylogenetic analysis. Phylogenetic analyses were performed using the maximum-parsimony and distance program of the 
PAUP 4.1 software (Swofford, 1998). Distance matrix analyses were generated with the Kimura two-parameter model for multiple substitutions (Kimura, 1980). Bootstrap values (Felsenstein, 1985) based on the analysis of 1000 replicates were determined to estimate the node reliability of the phylogenetic trees obtained by the parsimony, maximumlikelihood and distance methods.

The GenBank accession numbers of the $17 \mathrm{kDa}$ protein gene sequences are: Rickettsia australis, M74042 (unpublished), M28480 (Anderson \& Tzianabos, 1989); Rickettsia massiliae Mtu1 $^{\mathrm{T}}$, U11017 (unpublished); Rickettsia parkeri, U17008 (unpublished); Rickettsia rickettsii, M16486 (Anderson et al., 1987); R. typhi, M28481 (Anderson \& Tzianabos, 1989); and $R$. felis, AF195118 (this study). The GenBank accession numbers of all rickettsial $\operatorname{romp} A$ sequences compared are: Rickettsia aeschlimannii MC16 ${ }^{\mathrm{T}}$, U83446, U43800 (Fournier et al., 1998); Rickettsia africae ESF, U83436, U43790 (Fournier et al., 1998); Rickettsia akari Kaplan, L01461 (Gilmore, 1993); R. australis PHS, AF149108 (Stenos \& Walker, 2000); Rickettsia conorii Astrakhan, U83437, U43791 (Fournier et al., 1998); R. conorii Israeli, U43797, U83441 (Fournier et al., 1998); R. conorii Malish $7^{\mathrm{T}}$, U01028 (Crocquet-Valdes et al., 1994); R. conorii Moroccan, U83443, U43798 (Fournier et al., 1998); Rickettsia honei $\mathrm{RB}^{\mathrm{T}}$, AF018075, AF018076 (Stenos et al., 1998); Rickettsia japonica U43795, U83442 (Fournier et al., 1998); $R$. massiliae Bar29, U43792, U83444 (Fournier et al., 1998); $R$. massiliae Mtu1 ${ }^{\mathrm{T}}$, U83445, U43799 (Fournier et al., 1998); Rickettsia montanensis, U43801, U83447 (Fournier et al., 1998); R. parkeri, U43802, U83449 (Fournier et al., 1998); Rickettsia prowazekii, M28482 (Anderson \& Tzianabos, 1989); Rickettsia rhipicephali, U43803, U83450 (Fournier et al., 1998); $R$. rickettsii M31227 (Anderson et al., 1990); Rickettsia sibirica 246 ${ }^{\mathrm{T}}$, U43807, U83455 (Fournier et al., 1998); $R$. sibirica mongolotimonae, U43796, U83439 (Fournier et al., 1998); Rickettsia slovaca 13-B, U43808, U83454 (Fournier et al., 1998); and R. felis, AF191026 (this study).

Transmission electron microscopy. Adult fleas and larvae were anaesthetized on dry ice, their heads were removed and they were immediately dissected in a drop of fixative. Midguts with adjacent tissues were fixed in a mixture of $1.25 \%(\mathrm{v} / \mathrm{v})$ formaldehyde, $2.5 \%(\mathrm{v} / \mathrm{v})$ glutaraldehyde, $0.03 \%$ trinitrophenol and $0.03 \% \mathrm{CaCl}_{2}$ in $0.05 \mathrm{M}$ cacodylate buffer, $\mathrm{pH} 7 \cdot 3$ (Ito \& Rikihisa, 1981), post-fixed in $1 \% \mathrm{OsO}_{4}$ in the same buffer, stained en bloc with $1 \%$ uranyl acetate in $0 \cdot 1 \mathrm{M}$ maleate buffer ( $0 \cdot 1 \mathrm{M}$ maleic acid), $\mathrm{pH} 5 \cdot 2$, dehydrated in ethanol and embedded in Spurr low-viscosity epoxy resin (Polysciences). Ultrathin sections were cut on a Reichert Ultracut S ultramicrotome, stained with uranyl acetate and lead citrate, and examined in a Philips 201 electron microscope at $60 \mathrm{kV}$.

\section{RESULTS}

\section{Genetic analysis}

To verify that the DNA isolated from the fleas contained $R$. felis genomic DNA, RFLP analysis was performed on the $17 \mathrm{kDa}$ protein gene PCR product. The flea DNA extract amplified using rickettsial specific primers yielded a $434 \mathrm{bp}$ product. This product was then amplified by nested primers resulting in a 231 bp product. The nested product was then digested using restriction enzymes ( $A l u \mathrm{I}$ and $X b a \mathrm{I})$ and was resolved by agarose gel electrophoresis (data not shown). This method yielded a pattern that was characteristic of $R$. felis and was distinct from that of the $R$. typhi $17 \mathrm{kDa}$ protein gene PCR product (Schriefer et al., 1994a; Higgins et al., 1996). Once the DNA was confirmed to be that of $R$. felis, phylogenetic analysis using parsimony was conducted on the sequence (Table 2). Due to the limited number of $17 \mathrm{kDa}$ protein genes in GenBank, only nine Rickettsia species were analysed. Kimura two-parameter model analysis placed $R$. felis in the SFG of rickettsiae with only $5.3 \%$ divergence from $R$. australis. The $R$. felis $17 \mathrm{kDa}$ protein gene was only $5 \cdot 3-6 \cdot 6 \%$ divergent from the other SFG rickettsiae, but was 11.3 and $11.5 \%$ divergent from $R$. typhi and $R$. prowazekii, respectively.

Initially, the sequence was generated for the $R$. felis romp $A$ gene through the utilization of primers designed for the sequencing of the $R$. australis romp $A$ gene (Bouyer et al., 1999; Stenos \& Walker, 2000). This generated a $1279 \mathrm{bp}$ fragment. The amplification strategy for the rest of the R. felis romp $A$ gene involved the use of published primers that were shown to have been effective for delineating other rickettsial romp $A$ genes (Regnery et al., 1991; Walker et al., 1995). A set of primers was also developed by this laboratory from the published $R$. rickettsii romp $A$ sequence (Anderson et al., 1990) and from the generated $R$. felis sequence. A region of the $R$. felis romp $A$ gene sequence that was difficult to amplify was obtained by genome walking. This sequencing strategy yielded a DNA sequence of $5513 \mathrm{bp}$ with a $\mathrm{G}+\mathrm{C}$ content of $39.49 \mathrm{~mol} \%$ (data not shown). The gene had an ORF of $1860 \mathrm{bp}(\mathrm{G}+\mathrm{C}$ content of $40.27 \mathrm{~mol} \%$ ) that was found to contain four repeat units, which consisted of two complete repeat units (225 and $216 \mathrm{bp}$ ) and two repeat units containing deletions of 6 and $69 \mathrm{bp}$, resulting in altered units of 219 and $147 \mathrm{bp}$ in size, respectively. The promoter region of the $R$. felis romp $A$ gene was found to be similar to that of the $R$. australis romp $A$ gene (Stenos \& Walker, 2000). The coding sequences for the putative ribosome-binding site, -10 sequence and -35 region shared $100 \%$ homology between those two species. Genetic analysis of the full length $R$. felis romp $A$ gene ORF (1860 bp) and its comparison with other SFG romp $A$ gene ORFs that have been sequenced was problematic due to the fact that the $R$. felis gene contained a premature stop codon, thereby resulting in a much smaller coding region than $R$. australis $(6320$ $\mathrm{bp}), R$. conorii strain Malish $7^{\mathrm{T}}(6065 \mathrm{bp})$ and $R$. rickettsii (6749 bp). The $R$. felis romp $A$ gene ORF was found to have $51 \cdot 6,48 \cdot 6$ and $37.9 \%$ similarity to $R$. rickettsii, $R$. conorii and $R$. australis, respectively (data not shown). The marked divergence from $R$. australis is explained by its having the most divergent of rickettsial romp $A$ repeat domains (Stenos \& Walker, 2000). It was determined that the most effective means to analyse the $R$. felis romp $A$ gene would be to convert the gene into protein domains and combine the areas of interest outside of the repeats. The rickettsial rOmpA proteins were divided using the pattern 
Table 2 Kimura two-parameter model of divergence of 17 kDa protein gene of Rickettsia species

\begin{tabular}{|c|c|c|c|c|c|c|c|c|}
\hline Species & R. felis & R. typhi & R. conorii & R. montanensis & R. parkeri & R. prowazekii & R. rhipicephali & R. rickettsii \\
\hline \multicolumn{9}{|l|}{ R. felis } \\
\hline R. typhi & $0 \cdot 113262$ & & & & & & & \\
\hline R. conorii & $0 \cdot 058047$ & $0 \cdot 109749$ & & & & & & \\
\hline R. montanensis & $0 \cdot 064669$ & $0 \cdot 113315$ & $0 \cdot 018914$ & & & & & \\
\hline R. parkeri & $0 \cdot 066177$ & $0 \cdot 121191$ & $0 \cdot 008193$ & $0 \cdot 014556$ & & & & \\
\hline R. prowazekii & $0 \cdot 115637$ & $0 \cdot 05131$ & $0 \cdot 114369$ & $0 \cdot 11255$ & $0 \cdot 122627$ & & & \\
\hline R. rhipicephali & $0 \cdot 06216$ & $0 \cdot 115125$ & $0 \cdot 02068$ & $0 \cdot 012448$ & $0 \cdot 016631$ & $0 \cdot 12291$ & & \\
\hline R. rickettsii & $0 \cdot 061808$ & $0 \cdot 113979$ & $0 \cdot 00777$ & $0 \cdot 022668$ & $0 \cdot 011931$ & $0 \cdot 118665$ & $0 \cdot 024479$ & \\
\hline R. australis & $0 \cdot 053777$ & $0 \cdot 114894$ & $0 \cdot 039453$ & $0 \cdot 039664$ & $0 \cdot 041584$ & $0 \cdot 122358$ & $0 \cdot 037438$ & $0 \cdot 043379$ \\
\hline
\end{tabular}

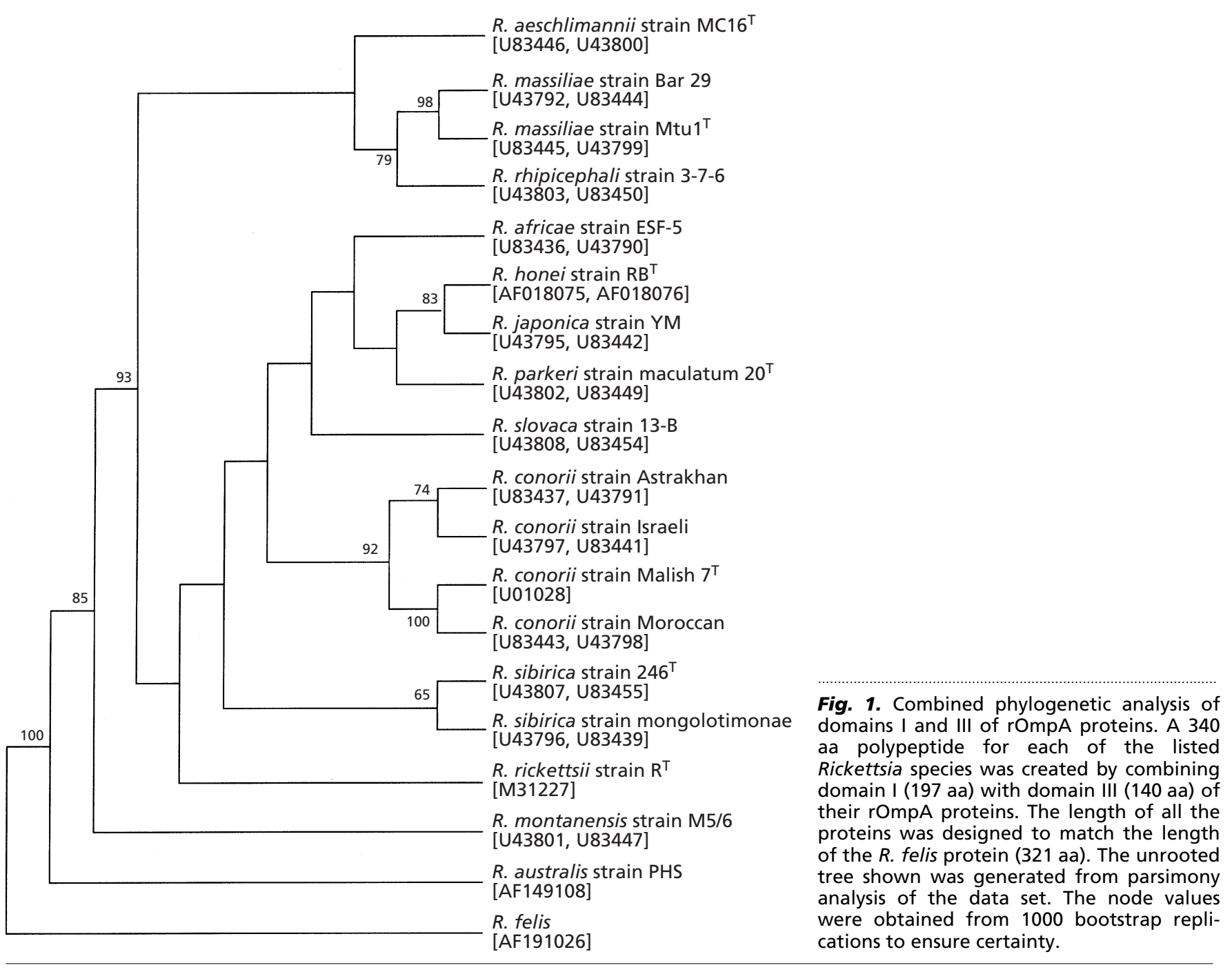

suggested by Anderson et al. (1990). The domains were linked using the method of Fournier (1998). Domain I of each rOmpA protein started at the initial methionine (residue 1) of the ORF and ended at the beginning of the repeat region. The domain I-domain III rOmpA fusions were 340 aa residues in length, which corresponds with the size of the protein that would be encoded by $R$. felis minus the repeat domain. This approach allowed the protein segments analysed to be closer in size: $R$. felis domain I being 206 aa residues in length; $R$. rickettsii and $R$. conorii, 211 aa residues; and $R$. australis, 265 aa residues. The entire repeat region of each of the rOmpA proteins was considered as domain II. The hydrophobic region that immedi- 


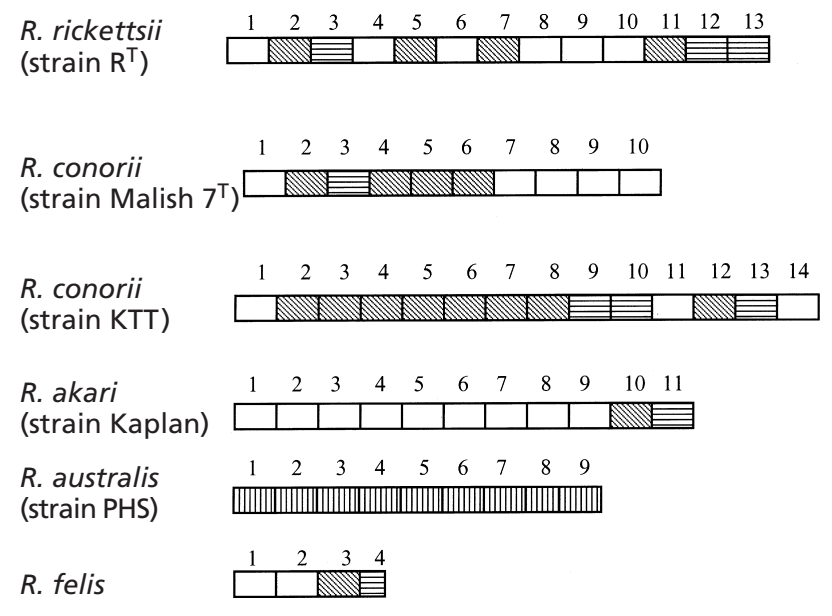

Fig. 2. Comparison of arrangement and length of sequenced Rickettsia species rOmpA repeat regions (domain II). White squares represent type I repeats $(75 \mathrm{aa})$, slanted lines represent type Ila repeats ( $72 \mathrm{aa})$, horizontal lines represent type IIb repeats $(72 \mathrm{aa})$ and vertical lines represent type III repeats ( 85 aa), which thus far have been observed only in $R$. australis. Domain II of $R$. felis is smaller in length than other Rickettsia species domain II.

ately follows the repeat domain is domain III. This region contained the premature stop codon in the $R$. felis romp A. Domain IV of the $R$. felis rOmpA, which was not used in our analysis because it contained several stop codons, consisted of primarily hydrophilic regions.

Amino acid sequences of $R$. felis rOmpA were analysed for percentage similarity utilizing the CLUSTAL algorithm (Higgins \& Sharp, 1989). Comparison of the R. felis rOmpA domain I with that of other Rickettsia species showed $36.4 \%$ similarity with $R$. australis, $40 \cdot 8 \%$ similarity with $R$. conorii (strain Malish $7^{\mathrm{T}}$ ) and $41.7 \%$ similarity with $R$. rickettsii at the amino acid level. Phylogenetic trees constructed from parsimony and distance analysis both indicated that $R$. felis was nearer to $R$. australis (Fig. 1). This was confirmed by the Kimura two-parameter model (Kimura, 1980; data not shown).

The repeat region (domain II) of the $R$. felis rOmpA is unique by several criteria when compared to other rOmpA repeat domains (Fig. 2). The repeat region is smaller than in other published naturally occurring species as it contains only three complete or nearcomplete repeat units and one partial repeat unit. The first repeat is a type I. The second repeat unit is homologous to a type I repeat with the deletion of 2 aa being the only difference. The third repeat is of type IIa and is 72 aa. Comparison of the partial fourth repeat to a type IIb unit showed that it had a deletion of 23 aa. Domain III of the $R$. felis rOmpA protein is most similar to $R$. australis ( $46.2 \%$; data not shown).

Recently in a study conducted by this laboratory (Moron et al., 2001), phylogenetic analysis (parsimony,
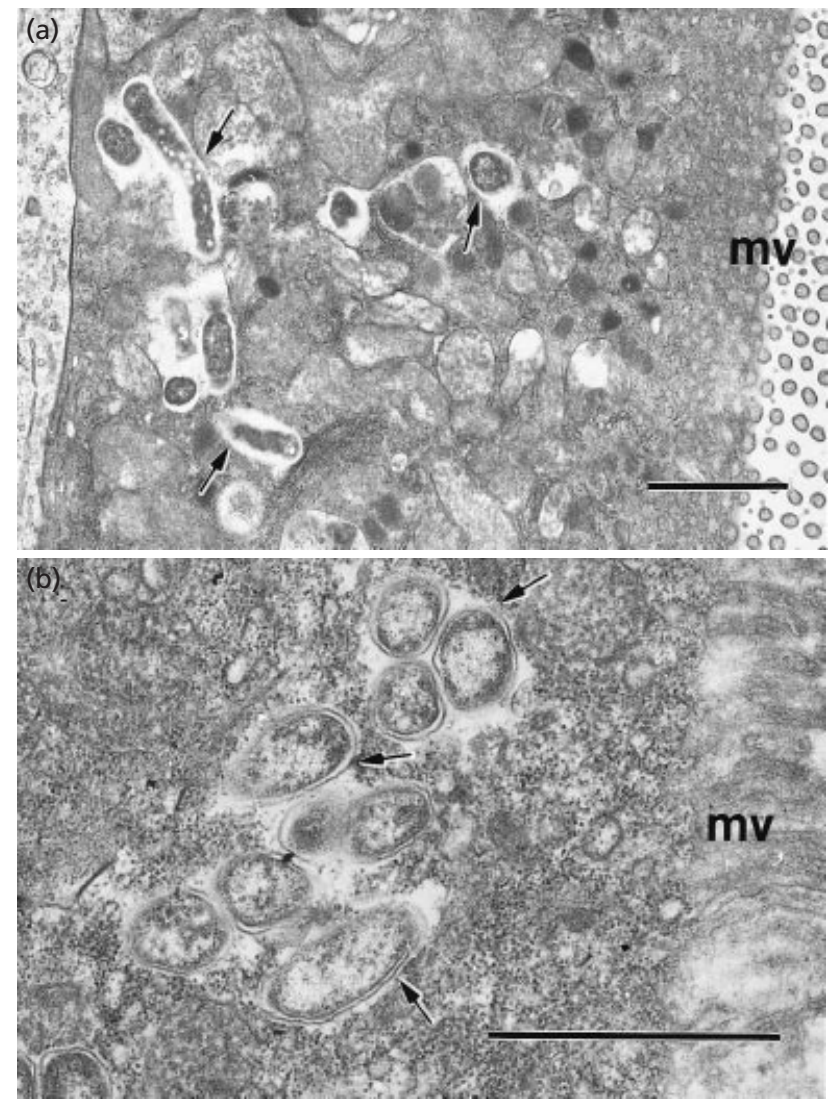

Fig. 3. Rickettsia felis in midgut epithelial cells of the cat flea. Bars, $1 \mu \mathrm{m}$. (a) In midgut epithelial cells of larvae, rickettsiae with dense cytoplasm (arrows) are located free in the cytosol surrounded by a clear space. Some rickettsiae have intracytoplasmic vacuoles. mv, Epithelial cell microvilli. (b) In a midgut epithelial cell of an adult flea, rickettsiae surrounded by two trilaminar membranes (arrows) are typically localized free in the cytosol. mv, Microvilli.

distance and maximum-likelihood) of the DNA sequences of rickettsial romp $B$ genes was performed. The $R$. felis rompB sequence was found to be less divergent from the SFG rickettsiae $(10-13 \%$ divergence) than from the typhus group (TG) rickettsiae $(18 \%)$. Analysis of the $R$. felis rOmpB protein by the CLUSTAL algorithm confirmed that $R$. felis is most similar to the SFG rickettsiae.

\section{Electron microscopy}

In adult cat fleas, rickettsiae were found in midgut epithelial cells and in underlying tissues, including muscles, and in oocytes. In larvae, rickettsiae were mostly localized in midgut epithelial cells. Rickettsiae were typically located free in the cytosol surrounded by electron-lucent clear spaces (Fig. 3). They varied in length, width and density of the cytoplasm, some rickettsiae in larvae having dense cytoplasm (Fig. 3a). Also, rickettsiae in larvae were observed to have intracytoplasmic vacuoles (Fig. 3a). 


\section{DISCUSSION}

Traditionally, organisms of Rickettsia species are propagated and analysed by microimmunofluorescence serotyping, $\mathrm{mAb}$ serotyping and comparison of proteins by SDS-PAGE and Western immunoblot comparison (Beati et al., 1992, 1997). Although effective, the serotyping methods are cumbersome and based on limited epitopes that are determined by small portions of the genome. Problems can also arise with the SDS-PAGE and Western immunoblot methods because the protein profiles of particular strains of a species can differ greatly (Walker et al., 1992). Rickettsial species, such as Rickettsia peacockii and Ehrlichia ewingii, that have thus far proven to be resistant to cultivation, have been classified by molecular analysis of selected genes (Anderson et al., 1987; Neibylski et al., 1997). Since the reliable continuous cultivation of $R$. felis has yet to be reported, it is felt that genetic analysis would be the best method for its classification, as was done for $R$. peacockii and E. ewingii.

$R$. felis has traditionally been placed within the TG of Rickettsia on the basis of reactivity of $R$. felis antigens with antibodies to $R$. typhi and the previous association of the TG with insects and the SFG with acarines (Adams et al., 1990; Azad et al., 1992). In contrast, data from genetic studies would place $R$. felis in the SFG (Azad et al., 1992; Roux \& Raoult, 1995; Stothard \& Fuerst, 1995). For example, comparison of a $381 \mathrm{bp}$ fragment of the CS gene of $R$. felis and several other rickettsial species indicated that there were $24 \mathrm{bp}$ differences between $R$. felis and $R$. prowazekii. This was similar to the 25 bp difference between $R$. rickettsii and $R$. prowazekii (Higgins et al., 1996). Higgins and others also reported that there was a $32 \mathrm{bp}$ difference between $R$. felis and $R$. typhi. There was only an $11 \mathrm{bp}$ difference between $R$. prowazekii and $R$. typhi, both members of the $\mathrm{TG}$, indicating that $R$. felis is genetically a different species. The relatively small difference, only $13 \mathrm{bp}$, between $R$. felis and R. rickettsii further supports the placement of $R$. felis in the SFG. The use of the CS gene has been recognized as a useful tool for analysis of the genus Rickettsia (Roux et al., 1997). Phylogenetic analysis of the 16S rRNA gene of 14 rickettsial species indicated that $R$. felis forms a clade with $R$. akari and $R$. australis (Stothard \& Fuerst, 1995). R. felis is divergent from the aforementioned bacteria by only 1.2 and $0.4 \%$, respectively. Our analysis of the $R$. felis $17 \mathrm{kDa}$ protein gene also indicates that $R$. felis does not belong in the TG of rickettsiae. $R$. felis was found to have an $11 \%$ divergence from the TG, which corresponds with the $10-12 \%$ divergence between the SFG and TG. These results were confirmed by a comprehensive study involving molecular analysis of multiple genes of several Rickettsia species, including R. felis (Andersson $\&$ Andersson, 1999). Phylogenetic analysis of the predicted amino acid sequences of the fts $Y$, polA and dnaE gene products also showed that $R$. felis is a member of the SFG. It was interesting to note that the genes used for the construction of the aforementioned tree were neighbouring genes representing a segment of slightly more than $9000 \mathrm{bp}$ of the $R$. felis genome.

Recently, a dengue-like illness was described that was caused by an unknown SFG rickettsia (ZavalaVelazquez et al., 1996). Since it was suspected that the aetiological agent in those cases was perhaps $R$. felis, it was decided to investigate whether the bacterium harbours a gene that encodes rOmpA (Bouyer et al., 1999). This was significant because only members of the SFG have been found to contain rompA. The complete sequence was obtained and the $R$. felis romp $A$ gene was characterized, thus providing evidence that R. felis is a Rickettsia of the SFG. A DNA sequence of $R$. felis $5513 \mathrm{nt}$ in length contained sequences homologous to romp $A$ including an ORF of $1860 \mathrm{bp}$ which would encode a protein of a predicted size of $60.5 \mathrm{kDa}$. Although the premature stop codon resulted in an estimated size of the $R$. felis rOmpA much smaller than the observed and predicted sizes of the other known rickettsial rOmpA proteins, it is not a truly unexpected phenomenon. It has been reported that two SFG Rickettsia species, $R$. rhipicephali and $R$. sibirica, contained termination codons in the middle region of the met $K$ gene (Andersson \& Andersson, 1999). R. felis was also found to have a deletion of $3018 \mathrm{bp}$ within the met $K /$ dnaE intergenic region, which is substantially greater than that seen with other Rickettsia species and $50 \mathrm{bp}$ in the $\mathrm{fmt} / \mathrm{rrl}$ intergenic region (Andersson \& Andersson, 1999). This phenomenon of deletion of a gene segment was also observed in the $R$. felis romp $A$ repeat region. This region consisted of three complete or near-complete repeat units and one partial repeat, which makes the region smaller than that of the other SFG rickettsiae for which the sequencing of domain II has been reported, namely $R$. rickettsii (13 repeats) and $R$. conorii strain Malish $7^{\mathrm{T}}$ (10 repeats) and Kenya tick typhus strain (14 repeats), $R$. akari (11 repeats) and $R$. australis (9 repeats) (Fig. 2). Further analysis of the $R$. felis repeat units showed that two of the repeats (the second and fourth) contained a different number of amino acids than previously observed in a rickettsia (Anderson et al., 1990; Gilmore \& Hackstadt, 1991; Gilmore, 1993; Crocquet-Valdes et al., 1994). Type I repeats consisted of 75 aa; whereas, type II repeats contained 72 aa. The newly designated type III repeats, which are unique to $R$. australis, contained 85 aa (Stenos \& Walker, 2000). R. felis contained a repeat unit of 73 aa that, if not for a deletion of 2 aa, would have been a typical complete type I repeat. There has also been a report of the deletion of most of the repeat domain of the romp $A$ gene of a laboratory-passaged strain of $R$. rickettsii (Matsumoto et al., 1996). This strain was found to have only one repeat unit within the mutated repeat domain instead of the expected 13 repeat units. Any influence that the aforementioned variations may have exerted upon serological assays is unknown at this time but is currently being studied by our group.

The evidence that $R$. felis contained a gene that encodes 
rOmpA suggests that $R$. felis is a novel Rickettsia species in the SFG. As stated previously, rOmpA distinguishes members of the SFG from TG rickettsiae, and the very presence of the gene in $R$. felis indicates that its current classification should be revised. In addition our phylogenetic analysis of the merged rOmpA proteins from 19 species of Rickettsia places $R$. felis near $R$. australis.

Although the existence of the $R$. felis rOmpA is of importance, we are left to ponder the reactivity of $R$. felis with antibodies stimulated by $R$. typhi (Azad et al., 1992). It could be debated that the first identification of $R$. felis in the cat flea was in fact $R$. typhi, but this seems unlikely as shown by the distinct RFLP pattern of the R. felis CS and $17 \mathrm{kDa}$ protein genes and their DNA sequences (Higgins et al., 1996; Azad et al., 1992). Perhaps a more plausible theory is that $R$. felis is closer to the common Rickettsia ancestor on the evolutionary scale than any of the previously analysed species. This theory could be supported by $R$. felis containing features of both the SFG and TG and also the observations that several genes of $R$. felis, including $\operatorname{romp} A$, have been found to contain large deletions of nucleotides. This is one of the first reports of a potentially dying virulence gene in the Rickettsia species.

The expression of the $R$. felis romp $A$ gene product is currently being investigated by our laboratory. Ultimately, the analysis of $R$. felis will allow the solution of several evolutionary questions. It is proposed that this bacterium, formerly known as the ELB agent, be designated Rickettsia felis sp. nov., type strain Ctenocephalides felis-LSU ${ }^{\mathrm{T}}$, and be placed in the SFG of rickettsiae. The type strain is maintained in an infected flea colony by the Department of Entomology, Louisiana State University Agricultural Center.

\section{Description of Rickettsia felis sp. nov.}

Rickettsia felis [felis. L. gen. n. felis of the cat) is named to recognize the discovery and origin of the bacterium in the cat flea (Ctenocephalides felis), a parasite of the cat (Felis domesticus) and opossum (Didelphis marsupialis) (Adams et al., 1990; Schriefer et al., 1994; Higgins et al., 1996; Matthewman et al., 1997].

The bacterium was first observed by electron microscopy of tissues of the cat flea and was reported to have the typical rickettsial membrane ultrastructure (Adams et al., 1990). The organism is $0.25-0.45 \mu \mathrm{m}$ in diameter by $1.5 \mu \mathrm{m}$ in length and has been observed by this laboratory in both adult fleas and their larvae (Fig. $3 a, b)$. The organism is maintained in the flea by vertical (transovarial) transmission (Azad et al., 1992). At the time of writing, reliable propagation of $R$. felis in culture has not been reported.

\section{ACKNOWLEDGEMENTS}

The authors wish to thank: Earl Andress for assistance with the flea colony; Bill Sweeney and the UTMB Protein Chemistry Laboratory for their assistance in the automated
DNA sequencing; Violet Han for expert assistance in electron microscopy; Ann Powers for advice in the phylogenetic analysis; Josie Ramirez-Kim and Kelly Cassity for their expert secretarial assistance in the preparation of this manuscript. The research was supported by grants from the National Institute of Allergy and Infectious Diseases (AI21242 and AI31431) and the National Institutes of Health (D43 TW00903).

\section{REFERENCES}

Adams, J. R., Schmidtmann, E. T. \& Azad, A. F. (1990). Infection of colonized cat fleas, Ctenocephalides felis (Bouche), with a rickettsia-like microorganism. Am J Trop Med Hyg 43, 400-409.

Anderson, B. E. \& Tzianabos, T. (1989). Comparative sequence analysis of a genus-common rickettsial antigen gene. $J$ Bacteriol 171, 5199-5201.

Anderson, B. E., Regnery, R. L., Carlone, G. M., Tzianabos, T., McDade, J. E., Fu, Z. Y. \& Bellini, W. J. (1987). Sequence analysis of the 17-kilodalton-antigen gene from Rickettsia rickettsii. $J$ Bacteriol 169, 2385-2390.

Anderson, B. E., McDonald, G. A., Jones, D. C. \& Regnery, R. L. (1990). A protective protein antigen of Rickettsia rickettsii has tandemly repeated near-identical sequences. Infect Immun 58, 2760-2769.

Anderson, B. E., Greene, C. E., Jones, D. C. \& Dawson, J. E. (1992). Ehrlichia ewingii sp nov., the etiologic agent of canine granulocytic ehrlichosis. Int J Syst Bacteriol 42, 299-302.

Andersson, J. O. \& Andersson, S. G. E. (1999). Genome degradation is an ongoing process in Rickettsia. Mol Biol Evol 16, 1178-1191.

Andersson, S. G. E., Stothard, D. R., Fuerst, P. A. \& Kurland, C. G. (1999). Molecular phylogeny and rearrangement of rRNA genes in Rickettsia species. Mol Biol Evol 16, 987-995.

Azad, A. F., Sacci, J. B., Nelson, W. M., Dasch, G. A., Schmidtmann, E. T. \& Carl, M. (1992). Genetic characterization and transovarial transmission of a typhus-like rickettsia found in cat fleas. Proc Natl Acad Sci US A 89, 43-46.

Beati, L., Finidori, J. P., Gilot, B. \& Raoult, D. (1992). Comparison of serologic typing, sodium dodecyl sulfate-polyacrylamide gel electrophoresis protein analysis, and genetic restriction length polymorphism analysis for identification of rickettsiae: characterization of two new rickettsial strains. J Clin Microbiol 30, 1922-1930.

Beati, L., Meskini, M., Thiers, B. \& Raoult, D. (1997). Rickettsia aeschlimannii sp. nov., a new spotted fever group rickettsia associated with Hyalomma marginatum ticks. Int J Syst Bacteriol 47, 548-554.

Bouyer, D. H., Stenos, J., Crocquet-Valdes, P., Foil, L. D. \& Walker, D. H. (1999). The identification and characterization of a previously undiscovered rompA-encoding gene in Rickettsia felis. In Rickettsiae and Rickettsial Diseases at the Turn of the Third Millennium, pp. 11-15. Edited by D. Raoult \& P. Brouqui. Paris, France: Elsevier.

Crocquet-Valdes, P. A., Weiss, K. \& Walker, D. H. (1994). Sequence analysis of the 190-kDa antigen-encoding gene of Rickettsia conorii (Malish 7 strain). Gene 140, 115-119.

Felsenstein, J. (1985). Confidence limits on phylogenies: an approach using the bootstrap. Evolution 39, 783-791.

Fournier, P.-E., Roux, V. \& Raoult, D. (1998). Phylogenetic analysis of spotted fever group rickettsiae by study of the outer surface protein rOmpA. Int J Syst Bacteriol 48, 839-849.

Gilmore, R. D., Jr (1993). Comparison of the romp $A$ gene repeat 
regions of Rickettsia reveals species-specific arrangements of individual repeating units. Gene 125, 97-102.

Gilmore, R. D., Jr \& Hackstadt, T. (1991). DNA polymorphism in the conserved $190 \mathrm{kDa}$ gene repeat region among the spotted fever group rickettsiae. Biochim Biophys Acta 1097, 77-80.

Henderson, G. \& Foil, L. D. (1993). Efficacy of diflubenzuron in simulated household and yard conditions against the cat flea Ctenocephalides felis (Bouche) (Siphonoptera: Pulicidae). $J$ Med Entomol 30, 619-621.

Higgins, D. G. \& Sharp, P. M. (1989). Fast and sensitive multiple sequence alignments on a microcomputer. CABIOS 5, 151-153.

Higgins, J. A., Radulovic, S., Schriefer, M. E. \& Azad, A. F. (1996). Rickettsia felis: a new species of pathogenic rickettsia isolated from cat fleas. J Clin Microbiol 34, 671-674.

Ito, S. \& Rikihisa, T. (1981). Techniques for electron microscopy of rickettsiae. In Rickettsiae and Rickettsial Diseases, pp. 213-227. Edited by W. Burgdorfer \& R. L. Anacker. New York: Academic Press.

Kimura, M. (1980). A simple method for estimating evolutionary rate of base substitutions through comparative studies of nucleotide sequences. J Mol Evol 16, 111-120.

Matsumoto, M., Tange, Y., Okada, T., Inoue, Y., Horiuchi, T., Kobayashi, Y. \& Fujita, S. (1996). Deletion in the $190 \mathrm{kDa}$ antigen gene repeat region of Rickettsia rickettsii. Microb Pathol 20 , 57-62.

Matthewman, L., Kelly, P., Hayter, D., Downie, S., Wray, K., Bryson, N., Rycroft, A. \& Raoult, D. (1997). Domestic cats as indicators of the presence of spotted fever and typhus group rickettsiae. Eur J Epidemiol 13, 109-111.

Moron, C. G., Bouyer, D. H., Yu, X.-J., Foil, L. D., Crocquet-Valdes, P. \& Walker, D. H. (2001). Phylogenetic analysis of the rompB genes of Rickettsia felis and Rickettsia prowazekii European human and North American flying squirrel strains. Am J Trop Med Hyg 62, 598-603.

Neibylski, M. L., Schrumpf, M. E., Burgdorfer, W., Fischer, E. R., Gage, K. L. \& Schwan, T. G. (1997). Rickettsia peacockii sp. nov., a new species infecting wood ticks, Dermancentor andersoni, in western Montana. Int J Syst Bacteriol 47, 446-452.

Noden, B. H., Radulovic, S., Higgins, J. A. \& Azad, A. F. (1998). Molecular identification of Rickettsia typhi and R. felis in coinfected Ctenocephalides felis (Siphonaptera: Pulicidae). J Med Entomol 35, 410-414.

Radulovic, S., Higgins, J. A., Jaworski, D. C. \& Azad, A. F. (1995a). In vitro and in vivo antibiotic susceptibilities of ELB rickettsiae. Antimicrob Agents Chemother 39, 2564-2566.

Radulovic, S., Higgins, J. A., Jaworski, D. C., Dasch, G. A. \& Azad, A. F. (1995b). Isolation, cultivation, and partial characterization of the ELB agent associated with cat fleas. Infect Immun 63, 4826-4829.

Regnery, R. L., Spruill, C. L. \& Plikaytis, B. D. (1991). Genotypic identification of rickettsiae and estimation of intraspecies sequence divergence for portions of two rickettsial genes. $J$ Bacteriol 173, 1576-1589.

Roux, V. \& Raoult, D. (1995). Phylogenetic analysis of the genus
Rickettsia by $16 \mathrm{~S}$ rDNA sequencing. Res Microbiol 146, 385-396.

Roux, V., Fournier, P. E. \& Raoult, D. (1996). Differentiation of spotted fever group rickettsiae by sequencing and analysis of restriction fragment length polymorphism of PCR-amplified DNA of the gene encoding the protein rOmpA. J Clin Microbiol 34, 2058-2065.

Roux, V., Rydkina, E., Eremeeva, M. \& Raoult, D. (1997). Citrate synthase gene comparison, a new tool for phylogenetic analysis, and its application for the rickettsiae. Int J Syst Bacteriol 47, 252-261.

Schriefer, M. E., Sacci, J. B., Dumler, J. S., Bullen, M. G. \& Azad, A. F. (1994a). Identification of a novel rickettsial infection in a patient diagnosed with murine typhus. J Clin Microbiol 32, 949-954.

Schriefer, M. E., Sacci, J. B., Jr, Taylor, J. P., Higgins, J. A. \& Azad, A. F. (1994b). Murine typhus: Updated roles of multiple urban components and a second typhus-like Rickettsia. J Med Entomol 31, 681-685.

Stenos, J. \& Walker, D. H. (2000). The rickettsial outer-membrane protein A and B genes of Rickettsia australis, the most divergent rickettsia of the spotted fever group. Int J Syst Evol Microbiol 50, 1775-1779.

Stenos, J., Roux, V., Walker, D. H. \& Raoult, D. (1998). Rickettsia honei sp. nov., the aetiological agent of Flinders Island spotted fever in Australia. Int J Syst Bacteriol 48, 1399-1404.

Stothard, D. R. \& Fuerst, P. A. (1995). Evolutionary analysis of the spotted fever and typhus group of rickettsia using $16 \mathrm{~S}$ rRNA gene sequences. Syst Appl Microbiol 18, 52-61.

Swofford, D. L. (1998). PAUP: phylogenetic analysis using parsimony (and other methods). version 4. Sunderland, MA: Sinauer Associates.

Walker, D. H., Liu, Q. H., Yu, X. J., Li, H., Taylor, C. \& Feng, H.-M. (1992). Antigenic diversity of Rickettsia conorii. Am J Trop Med Hyg 47, 78-86.

Walker, D. H., Feng, H. M., Saada, J. I., Crocquet-Valdes, P. A., Radulovic, S., Popov, V. L. \& Manor, E. (1995). Comparative antigenic analysis of spotted fever group rickettsiae from Israel and other closely related organisms. Am J Trop Med Hyg 52, 569-576.

Webb, L., Carl, M., Malloy, D. C., Dasch, G. A. \& Azad, A. F. (1990). Detection of murine typhus infection in fleas by using the polymerase chain reaction. J Clin Microbiol 28, 530-534.

Williams, S. G., Sacci, J. B., Jr, Schriefer, M. E., Anderson, E. M., Fujioka, K. K., Sorvillo, F. J., Barr, A. R. \& Azad, A. F. (1992). Typhus and typhus-like rickettsiae associated with opossums and their fleas in Los Angeles County, CA. J Clin Microbiol 30, 1758-1762.

Zavala-Velazquez, J. E., Yu, X.-J. \& Walker, D. H. (1996). Unrecognized spotted fever group rickettsiosis masquerading as dengue fever in Mexico. Am J Trop Med Hyg 55, 157-159.

Zavala-Velazquez, J. E., Sosa-Ruiz, J. A., Sanchez-Elias, R. A., Becessa-Carmona, G. \& Walker, D. H. (2000). Rickettsia felis rickettsiosis in Yucatan. Lancet 356, 1079-1080. 